Dossiê: Desigualdades, estratificação e justiça social

ENCARTE DIGITAL

\title{
A desigualdade no "topo" \\ Empregadores negros e brancos no mercado de trabalho brasileiro
}

\author{
Inequality on the top \\ Black and white employers in Brazilian job market
}

\author{
Jordão Horta Nunes* \\ Neville Julio de Vilasboas e Santos**
}

\begin{abstract}
Resumo: Os empregadores, em razão de sua posição no binômio capital-trabalho, ocupariam provavelmente estratos de remuneração mais alta. No entanto, há desníveis de rendimentos, como ocorre entre trabalhadores brancos e negros, em que estes se tornam empregadores preponderantemente em setores menos privilegiados e ocupações com alto índice de informalidade, em pequenos empreendimentos com menor estabilidade. Este artigo a) apresenta um breve perfil de empregadores negros e brancos no Brasil; b) demonstra as desigualdades de inserção na estrutura do mercado de trabalho e c) mensura os diferenciais médios de renda a fim de determinar o peso de fatores produtivos e da discriminação racial sobre a renda média dos empregadores estratificados por raça e sexo. Com base na análise quantitativa, empregando o método de decomposição de Oaxaca-Blinder, sustenta-se que existe uma diferença significativa de rendimentos entre estratos orientados por raça e sexo de empregadores que não pode ser creditada unicamente a fatores associados, como nível de escolaridade, experiência ou diferentes inserções ocupacionais, mas provém de um resíduo não explicado que é atribuído à discriminação de raça ou sexo.
\end{abstract}

Palavras-chave: Desigualdade. Rendimento. Empregador. Raça. Sexo.

* Doutor em Sociologia pela Universidade de São Paulo (USP, São Paulo, Brasil) e professor na Faculdade de Ciências Sociais da Universidade Federal de Goiás em Goiânia, GO, Brasil, onde atua na área de sociologia do trabalho, metodologia das ciências sociais e estudos na área de trabalho e gênero<jordao_fcs@ufg.br>.

**Doutor em Sociologia pela Universidade Federal de Goiás (UFG, Goiás, Brasil) e professor do Instituto Federal de Educação, Ciência e Tecnologia de Goiás, campus Anápolis, GO, Brasil. Atua nas áreas de desigualdades raciais, estratificação social, metodologia das ciências sociais, trabalho e gênero<nevilleifg@gmail.com>.

Este artigo tem como base uma comunicação apresentada no GT Trabalho e ação sindical na sociedade contemporânea, durante o 39을 Encontro da Anpocs, em outubro de 2015. O texto foi atualizado e reformulado com base nas considerações de colegas presentes e nos requisitos para publicação neste periódico.

Civitas, Porto Alegre, v. 16, n. 2, e87-e109, abr.-jun. 2016 


\begin{abstract}
The employers, due to their position on the capital-labour ratio, would probably occupy high-income strata. However, there are income gaps, as occurs with wage in black and white workers, where the former become employers in traditionally less privileged sectors and occupations with high levels of informality, in small ventures which with lower stability. This article: a) presents a brief profile of black and white employers in Brazil; b) demonstrate the insertion inequalities in the job market structure; c) measure the average income differentials in order to determine the weight of productive factors and race or sex-based discrimination over employer's average incomes. Based on quantitative analytic models, as the Blinder-Oaxaca decomposition method, we argue there is a significant wage gap between race and sex groups of Brazilian employers that cannot be credited only to associated factors like educational level, tenure or different occupation locations, but comes otherwise of an unexplained residue attributed to race and sex discrimination.
\end{abstract}

Keywords: Inequality. Income. Employer. Race. Sex.

\title{
Introdução
}

As desigualdades, nas ciências sociais, são tratadas como processos de produção e distribuição de bens e recursos, materiais e simbólicos, escassos na sociedade (Grusky, 2001). A análise desses processos pode se dar de duas formas: a) exame das causas, estruturas e usos da desigualdade; e b) análise das consequências da distribuição desigual de oportunidades e recompensas. $\mathrm{Na}$ mediação entre essas duas formas existem desigualdades adscritas, que não se relacionam diretamente com as diferenças de performances, mas são desvantagens produzidas historicamente entre grupos sociais baseadas na ideia de diferença étnica/racial e sexual (Lima, 2012). Segundo Tilly (1999), as desigualdades adscritas são duráveis porque têm um caráter substantivo, uma vez que não envolvem critérios de aquisição de capacidades. Daí decorre seu poder de tornarem-se preditoras das chances de sucesso dos indivíduos (Therborn, 2006; Grusky, 2001).

É possível identificar nos estudos sobre estratificação e desigualdade social quatro eixos principais em torno dos quais se constituem e se expressam as hierarquias sociais: a) as relações sociais de produção; b) a estrutura ocupacional; c) a distribuição de rendimentos; e d) as diferenças no estilo de vida e nos padrões de distinção social. Cada um desses eixos corresponde a tradições teóricas específicas, que se constituíram a partir das influências das abordagens clássicas de autores como Marx, Weber, Durkheim, Ricardo e, mais recentemente, Bourdieu (Wright, 2005). Além disso, critérios adscritos como raça e sexo se sobrepõem a esses quatro eixos, criando padrões de distribuição de bens e recursos que combinam características adquiridas ao longo da vida e características ditas "inatas". Embora esse campo de pesquisa 
tenha se fundamentado com base no destaque às diferenças de perspectivas teóricas, o grande desafio na atualidade é combinar na análise da desigualdade e da estratificação contribuições conjugadas de diferentes eixos, levando em conta suas limitações e possibilidades.

O interesse deste artigo é explicar a que se deve a desigualdade de rendimentos verificada no interior do grupo dos que se declaram empregadores na Pesquisa Nacional por Amostra de Domicílio (Pnad) de 2014, do Instituto Brasileiro de Geografia e Estatística (IBGE). Esse interesse exige a articulação entre os três primeiros eixos acima citados, considerando que: a) os empregadores constituem um grupo específico do ponto de vista das relações sociais de produção, pois se destacam pela posse dos meios de produção; b) ainda que sejam igualmente proprietários dos meios de produção, se diferenciam pelo grupo ocupacional ao qual pertencem e atividade econômica que desempenham; e c) se diferenciam também pelo nível de rendimentos que auferem.

Portanto, o argumento aqui apresentado parte da constatação de que embora seja possível considerar os empregadores como um grupo homogêneo com relação a um dos eixos de estratificação, não é possível afirmar o mesmo em relação aos outros eixos, pois as desigualdades e hierarquias sociais que decorrem dos diferentes eixos de estratificação não se sobrepõem. Isso torna complexa a tarefa de compreender a estratificação dentro de um grupo que se caracteriza por um elemento comum, mas difere com relação a outros elementos. Interessa saber em que medida características adscritas como raça e sexo interferem nesse processo de diferenciação segundo eixos de estratificação diversos.

O objetivo específico deste artigo, portanto, é mensurar os diferenciais médios entre os rendimentos dos/as empregadores/as negros/as e os rendimentos dos/as empregadores/as brancos/as, a fim de determinar o peso de fatores produtivos e da discriminação racial e sexual sobre a renda média. ${ }^{1}$

\section{Raça, sexo, renda e estratificação social}

Nas três últimas décadas do século 20 diversos pesquisadores passaram a questionar as antigas teses de que a modernização política e econômica, com uma consequente distribuição mais equitativa dos recursos e oportunidades,

\footnotetext{
${ }^{1}$ Discriminação racial é empregada tanto como ação ou comportamento direto e explícito de inferiorização e negação do outro, quanto no sentido de preterição ou discriminação velada, que utiliza por vezes o próprio silêncio ou outra estratégia sutil para impedir, negar ou dificultar o acesso do outro a determinados bens materiais ou simbólicos. Essa segunda acepção é a mais adequada à perspectiva adotada neste artigo.
} 
viria a extinguir a desigualdade racial remanescente do Brasil colonial e fazer vigorar os critérios de classificação puramente econômicos de uma "moderna" sociedade de classes. Surgiram, contudo, demonstrações empíricas da existência e da persistência da discriminação racial no acesso a oportunidades de mobilidade social (ver Hasenbalg, 1979; Hasenbalg e Silva, 1988; Hasenbalg, Silva e Lima, 1999).

Os estudos quantitativos sobre a inserção no mercado de trabalho no Brasil demonstraram que os negros estão sempre em desvantagem em relação aos brancos nas chances de mobilidade social, pois estão mais submetidos a um "ciclo de desvantagens acumulativas", que remete às desigualdades presentes na origem social ou, mais precisamente, na ocupação e nível educacional dos pais, e que permanecem nas esferas da educação e do trabalho nas trajetórias de vida dos negros (Hasenbalg e Silva, 1998; 2003). Outro fator componente das desvantagens acumulativas é a distribuição geográfica desigual de grupos de cor no território brasileiro, com os brancos ocupando majoritariamente o sul e o sudeste e os negros as regiões historicamente menos desenvolvidas do ponto de vista econômico, numa distribuição resultante da dinâmica da escravidão no Brasil e da política de migração europeia que introduziu os trabalhadores brancos europeus principalmente no sul e sudeste.

Para Tilly (1999), a família e o sistema escolar se interpõem entre as diferentes posições na estrutura de classes e as oportunidades padronizadas de mobilidade social relativas a elas. Além dos efeitos de características tais como raça e sexo, a família e a educação ajudam a produzir e distribuir características - habilidades técnicas e cognitivas, traços de personalidade, modos de autoapresentação e credenciais - que o mercado de trabalho converte em desigualdade de renda e hierarquias ocupacionais. Sob a ideologia da igualdade de oportunidades, potencializada pela ampliação do sistema de ensino, tal processo reproduz a divisão social do trabalho disfarçando o grau em que as posições de classe são transmitidas de geração em geração (Hasenbalg e Silva, 2003).

A principal tese que deriva desses estudos é que o preconceito e a discriminação raciais estão intimamente associados à competição por posições na estrutura social, e produzem diferenças entre os grupos de cor na apropriação de posições na hierarquia social. Tais estudos têm apontado que, independente da raça, há uma forte rigidez na estrutura social brasileira. Essa rigidez se apresenta como racial na tentativa de aquisição ou manutenção de status elevado, nas chances de mobilidade ascendente, na maior desigualdade racial entre os mais escolarizados e mais ricos, em posições ocupacionais de 
maior status e na maior probabilidade de perder posição social (Osório, 2004, 2009; Santos, 2005; Ribeiro, 2009; Lima, 2012; Rocha, 2015).

As pesquisas recentes têm mostrado que, em condições de extrema pobreza, o atributo racial se destaca pouco, exceto em relação ao desemprego, o que indica que a cor consiste em um estigma na busca pelo emprego. A variável sexo, por sua vez, tem demonstrado força tanto entre os pobres quanto entre os ricos. Quanto mais rico é o contexto investigado, maior o peso do atributo racial na explicação das disparidades entre indivíduos negros e brancos. Desse modo, a raça reflete tanto posições individuais de status definido pela cor da pele - como também redes de relações na sociedade, configurando-se como uma variável estrutural (Lima, 2001). Isto indica quão importante é investigar as desigualdades de raça e sexo entre os empregadores.

Uma das formas de manifestação das desigualdades de raça e sexo é a desigualdade de renda. Importantes diferenciais de rendimento separam negros e brancos, homens e mulheres. Ao afirmar isto, não se assume aqui que a renda é a única ou mais importante variável dependente que indica a desigualdade. No entanto, o que confere à renda um caráter explicativo interessante é o seu poder de se converter em vários outros bens sociais, sejam materiais ou não.

Segundo Erikson e Goldthorpe (2002), até recentemente os sociólogos se interessavam quase exclusivamente pela classe, em detrimento da renda. O interesse pela classe e pela mobilidade de classe se dá não apenas como variável dependente (a ser explicada), mas também como independente (variável explanatória), usando-a em concorrência com outras variáveis, incluindo a renda e a mobilidade de renda, em sua capacidade de influenciar a variação em um amplo espectro de chances de vida e escolhas de vida. É possível, para Erikson e Goldthorpe (2002) - que julgam as posições de classe como sendo determinadas pelas relações de trabalho - considerar a classe como uma boa proxy para o rendimento permanente. Além disso, seu poder explanatório reside no fato de que está apta a capturar importantes aspectos das relações sociais da vida econômica.

Entretanto, para Myles (2003), a renda é fundamental para a compreensão sociológica da desigualdade, pois alterações na distribuição de rendimentos tanto entre as posições de classe quanto dentro de uma mesma classe podem influenciar a distribuição de riqueza, poder e prestígio. Para este autor, o legado dos sociólogos do século 19, como herança da economia política, é o entendimento de que a estrutura da desigualdade é construída sobre a divisão do trabalho, como uma teoria da "estrutura dos espaços vazios". Essa formulação é falha, para Myles (2003), porque as mudanças parecem ocorrer com mais frequência e profundidade dentro das classes e grupos ocupacionais, 
e não entre eles. Em suma, as mudanças na estrutura da desigualdade de renda nem sempre estão relacionadas com as mudanças observadas na estrutura de classe (Myles, 2003).

Sorensen (2000), no esforço de retomar a análise de classe baseada em uma teoria da renda, se ocupou dos padrões contemporâneos de crescimento da desigualdade dentro das classes e grupos ocupacionais. Ele e outros autores demonstraram que se nos anos 1980 a desigualdade cresceu na base da distribuição, nos anos 1990 seu crescimento se concentrou no topo da distribuição (Card e Dinardo, 2002; Atkinson, 2003; Piketty e Saez, 2003; Diprete, 2007). Com base nas contribuições desses autores, é possível considerar a renda como uma variável dependente legítima e apta a ser utilizada como proxy da posição de classe, mas também - e principalmente - enquanto indicador de desigualdades dentro de uma mesma classe.

Ainda que haja uma pequena e lenta redução da desigualdade entre homens e mulheres no mercado de trabalho, ao associarmos sexo e cor fica nítida a hierarquia de rendimentos com homens brancos invariavelmente no topo, homens negros e mulheres brancas se alternando em posições intermediárias - a depender da ocupação - e mulheres negras com rendimentos significativamente menores. Mesmo diante da maior escolarização das mulheres brancas em relação aos homens brancos, e das mulheres negras em relação aos homens negros, os homens continuam auferindo as rendas mais altas (Soares, 2000; Guimarães, 2002; Santos, 2005, 2008, 2009).

\section{Renda, posição de classe e estratificação}

Relações de produção, ocupação, renda e estilo de vida são elementos fundamentais que cercam a discussão sobre "classe", conceito nuclear das principais teorias da estratificação social. Como não há uma correspondência direta entre esses elementos, não há um consenso em torno da operacionalização do conceito. Além disso, as pesquisas na área de sociologia têm demonstrado exaustivamente a importância da educação na definição das classes sociais (Soares, 2000; Medeiros, 2003; Osório, 2004; Ribeiro, 2009; Scalon, 2009; Rocha, 2015).

Wright e Perrone (1977) definem classe como um grupo social que tem uma relação comum com os meios de produção e se relaciona, por princípio, de maneira antagônica e contraditória com os grupos sociais que ocupam uma posição diferente perante a produção. Essa perspectiva permite compreender a classe a partir da extração de mais-valia e da exploração. Seria possível afirmar que a ocupação, nesse sentido, contribuiria mais para a definição de uma determinada "situação de classe", bem como a renda decorrente dessa 
ocupação contribuiria para a definição de uma "situação de mercado", que indicaria a distância social - em termos de condições de vida - entre os indivíduos, inclusive os pertencentes à "mesma classe". "Empregador", grupo social que aqui nos interessa, na abordagem de Wright (2005), corresponde a uma categoria de classe, cujos indivíduos se caracterizam por serem donos de ou possuírem direitos sobre ativos de produção, comprarem força de trabalho de outros e não venderem sua própria força de trabalho. Esta classe não contempla, na visão de Wright (2005), como contemplaria na visão de Dahrendorf (2014), os gerentes, executivos ou supervisores, que não possuem os meios de produção, apesar de controlarem a força de trabalho de outros, já que fazem isso vendendo sua força de trabalho para os empregadores. Os gerentes representam a emergência de uma estrutura de autoridade dentro da empresa capitalista que é parcialmente diferenciada da propriedade (Wright e Perrone, 1977).

A propriedade dos meios de produção constitui um complexo sistema de relações sociais, de realização de direitos e reivindicação de aparatos de produção. Assim, mantém-se a estrutura de classe e o antagonismo. Entretanto, isto não implica que os empregadores sejam uma categoria homogênea. Ao contrário, esse grupo comporta uma heterogeneidade muito grande, abarcando realidades empíricas muito distintas e, por vezes, contraditórias e difíceis de classificar nos parâmetros teoricamente definidos. Nos países em que o mercado de trabalho tem altos níveis de desregulamentação e informalidade, essa heterogeneidade parece ser ainda maior, o que coloca desafios muito complexos para a definição e operacionalização da categoria analítica de empregador. Assim como existem trabalhadores muito bem pagos e também trabalhadores muito pobres, altos gerentes e pequenos encarregados, existem capitalistas muito ricos e também os pequenos empregadores locais. Resta saber, no entanto, em que medida a existência de diferenças internas desafia a importância das classes como categorias estruturais.

Para Wright (1979), a posição de classe é uma variável crítica de mediação entre a origem social e a renda. Então, a posição de classe afeta não apenas a renda da posição ocupacional, mas também a extensão na qual as características de origem, elas mesmas, podem ser convertidas em renda. Em particular, a posição de classe influencia fortemente a extensão na qual a educação influencia a renda. A especificidade dos empregadores é que a sua renda, teoricamente, é consequência direta da quantidade de ativos de capital controlados pelo empregador, e apenas consequência indireta da educação do empregador. Portanto, a educação importará para a renda dos empregadores 
somente se existir uma forte relação entre a quantidade de propriedade e o nível de educação (Wright, 1977).

É importante ressaltar que o efeito mais significativo das categorias de raça e sexo é, sem dúvida, operar por meio de mecanismos que classificam as pessoas em várias categorias de classe, em primeiro lugar (Wright, 2002). Tanto homens negros quanto mulheres são sub-representados na categoria de empregador, e isto deve ser considerado na análise. Então, quando examinamos diferenças de renda nas categorias de raça e sexo entre empregadores, temos que considerar que essas diferenças ocorrem depois dos efeitos do racismo e do sexismo já terem operado para manter certas pessoas totalmente fora do mercado de trabalho, para evitar que algumas encontrassem empregos estáveis em tempo integral e para influenciar a distribuição de raça e sexo entre as classes, impedindo que parte dos homens negros, mulheres negras e mulheres brancas alcançassem a posição de empregador em função destas características adscritas.

Embora não caiba no escopo do presente artigo, é preciso considerar ainda que, mesmo após adentrar a posição social de empregador/a, negros/as e brancos/as não são distribuídos proporcionalmente dentro desta categoria de classe. Os/as negros/as não apenas estão sub-representados entre os empregadores, mas sua representação decresce ao longo dos centis de renda, ou seja, estão em número ainda menor entre empregadores/as com rendimentos maiores.

Tabela 1. Distribuição dos/as empregadores/as, segundo cor/raça, ao longo dos centis de rendimentos - Brasil 2014

\begin{tabular}{cccc}
\hline Centis & Brancos/as (\%) & Negros/as (\%) & Total $(\%)$ \\
\hline 1 & 42,74 & 57,29 & 100,0 \\
5 & 56,09 & 43,91 & 100,0 \\
10 & 47,76 & 52,24 & 100,0 \\
25 & 60,85 & 39,15 & 100,0 \\
50 & 66,00 & 34,00 & 100,0 \\
75 & 72,16 & 27,84 & 100,0 \\
90 & 75,11 & 24,89 & 100,0 \\
95 & 84,97 & 15,03 & 100,0 \\
99 & 79,36 & 20,64 & 100,0 \\
\hline
\end{tabular}

Fonte: Microdados Pnad (2014), IBGE. 
A tabela atrás demonstra nitidamente um padrão de distribuição por cor/raça que é desfavorável aos/as negros/as. Nos centis de renda mais baixos, sua representação chega a ser maior que a dos/as brancos/as $\left(1^{\circ} \mathrm{e}\right.$ $10^{\mathrm{O}}$ centis). Entretanto, do $25^{\circ}$ centil em diante a presença de negros/as cai progressivamente, para aumentar em 5 pontos percentuais apenas no último. Portanto, dizer que os/as negros/as estão sub-representados no grupo dos empregadores não é dizer tudo o que se pode sobre a desigualdade nesse grupo. Eles estão sub-representados especialmente nos estratos que obtêm maior renda e, por isso, não dispõem das mesmas condições que o grupo dos/as brancos/as. Essa tabela revela, portanto, a disparidade no interior de um grupo que, em princípio, poderia ser tomado como homogêneo do ponto de vista da posição na ocupação, mas que, na realidade, é internamente segmentado do ponto de vista ocupacional e hierarquizado do ponto de vista dos rendimentos. Tal composição racial do grupo empregador leva a crer que as disparidades de rendimento entre os grupos raciais cresçam ao longo da distribuição dos rendimentos.

As afirmações precedentes, contudo, se apresentam como hipóteses cuja verificação não cabe no teor deste artigo, que enfoca as disparidades em torno dos rendimentos médios, com o objetivo de demonstrar que a discriminação opera não somente entre os empregados, submetidos a uma lógica salarial, mas também no interior do grupo que emprega.

\section{Análise da decomposição}

O capital humano pode ser compreendido como o conjunto das habilidades individuais vinculadas à capacidade produtiva, e incorporadas na qualificação para determinadas atividades (Becker, 1962; Carvalho, Néri e Silva, 2006). Somente após 1950 as análises mais sofisticadas do investimento em educação e treinamento passaram a incluir a produtividade em suas discussões, iniciando uma exploração das implicações do investimento de capital humano nas questões relacionadas à produção.

A estratégia metodológica empregada nesta análise consiste em estimar a equação de salários (Mincer, 1974) e decompor o diferencial de salários através do método proposto por Oaxaca (1973). Entretanto, não se trata aqui de salários, mas dos rendimentos de empregadores, considerando a hipótese de que um diferencial de rendimentos em favor dos brancos pode estar, em parte, relacionado à discriminação presente no mercado de trabalho que faz com que o desempenho e os resultados de empregadores negros sejam inferiores, controlando um conjunto de variáveis que podemos chamar de "sistêmicas". Teoricamente, há razões para considerarmos que as variáveis que determinam 
os salários dos empregados também estejam relacionadas à determinação da renda dos empregadores, acrescida apenas de uma variável que indique o tamanho do estabelecimento produtivo mantido pelo empregador.

A teoria do capital humano aplicada à análise de regressão fornece um arcabouço para avaliar como características individuais influenciam nos retornos dos rendimentos. Os trabalhos de Becker (1962) e Mincer (1974) descrevem a ligação entre o ciclo da vida de um indivíduo e os investimentos em capital humano. Compreender os determinantes dos rendimentos dos empregadores a partir de suas características individuais e informações sobre o mercado de trabalho contribui para avançar no conhecimento das especificidades do mercado de trabalho. No caso brasileiro, bem como no de outros países capitalistas, é fundamental acrescentar à análise variáveis que permitam captar também os efeitos de padrões de relações sociais historicamente produzidas por clivagens de raça e sexo.

A técnica desenvolvida por Oaxaca (1973) e Blinder (1973) aplica-se à decomposição dos diferenciais de rendimento passíveis de serem observados pela equação minceriana. Tal método tem sido amplamente aplicado ao estudo do mercado de trabalho em diferentes países. O método divide os diferenciais de rendimentos entre dois grupos numa parte que é "explicada" por diferenças de características relevantes para o trabalho, como características produtivas dos indivíduos e características da estrutura do mercado de trabalho, e uma parte que não pode ser relacionada a tais características na determinação dos rendimentos. "Essa parte 'não explicada' é em geral usada como uma medida para discriminação, mas também subsume os efeitos das diferenças do grupo em preditores não observados" (Jann, 2008, p. 453).

Neste caso consideramos dois grupos, brancos (B) e negros $(\mathrm{N})$, uma variável de resultado $(\mathrm{R})$, rendimento médio, ${ }^{2}$ e um conjunto de variáveis preditoras, que podem ser agrupadas:

- características pessoais: sexo, escolaridade, ${ }^{3}$ idade, ${ }^{4}$ tempo de experiência; 5

\footnotetext{
${ }^{2}$ Expressa em logaritmos de rendimento por hora, para adequação ao cálculo de modelos loglineares, utilizados nos métodos de decomposição. A utilização do rendimento médio por hora é mais interessante que o rendimento mensal ou semanal, pois possibilita a comparação dos rendimentos de empregadores que cumprem diferentes jornadas de trabalho.

${ }^{3}$ Variável ordinal, v4803 da Pnad 2014, expressa por uma sequência numérica de valores correspondente aos níveis de escolaridade, desde "sem instrução e menos de um ano" até "15 ou mais" anos de estudo.

${ }^{4}$ Variável quantitativa discreta, v8005 da Pnad 2014, expressa em anos.

${ }_{5}^{5}$ Variável quantitativa discreta construída pela diferença entre anos de idade (variável 8005) e idade com que começou a trabalhar (v9892).
} 
- localização geográfica: remetendo às macrorregiões brasileiras; ${ }^{6}$

- características econômicas: número de empregados, ${ }^{7}$ atividade econômica. ${ }^{8}$

$\mathrm{O}$ modelo permite estimar o quanto da diferença das médias dos rendimentos $(\Delta)$, pode ser explicado pelas diferenças dos grupos nas variáveis preditoras, onde $\mathrm{E}(\mathrm{R})$ denota o valor esperado (expected value) do rendimento:

$$
\Delta=\mathrm{E}\left(\mathrm{R}_{\mathrm{B}}\right)-\mathrm{E}\left(\mathrm{R}_{\mathrm{N}}\right)
$$

Tomando como base o modelo linear $\mathrm{R}_{1}=\mathrm{X}_{1 \beta_{1}}^{\prime}+\varepsilon_{1}, \mathrm{E}\left(\varepsilon_{1}\right)=0,1 \in(\mathrm{B}, \mathrm{N})$, onde $\mathrm{X}$ é um vetor contendo os previsores e uma constante, $\beta$ contém os coeficientes angular e linear e $\varepsilon$ é o erro, a diferença de médias resultante pode ser expressa como as diferenças na previsão linear das médias relativas aos grupos dos regressores. Ou seja:

$$
\begin{aligned}
& \Delta=\mathrm{E}\left(\mathrm{R}_{\mathrm{B}}\right)-\mathrm{E}\left(\mathrm{R}_{\mathrm{N})}=\mathrm{E}\left(\mathrm{X}_{\mathrm{B}}\right)_{\mathrm{B}}^{\beta}-\mathrm{E}\left(\mathrm{X}_{\mathrm{N}}\right)_{\mathrm{N}}^{\beta},\right. \text { já que } \\
& \mathrm{E}\left(\mathrm{R}_{\mathrm{l}}\right)=\mathrm{E}\left(\mathrm{X}_{\mathrm{I}}^{\prime}+\varepsilon_{\mathrm{l}}\right)=\mathrm{E}\left(\mathrm{X}_{\mathrm{l}}^{\prime} \beta_{\mathrm{I}}\right)+\mathrm{E}\left(\varepsilon_{\mathrm{l}}\right)=\mathrm{E}\left(\left(\mathrm{X}_{\mathrm{l}}\right)^{\prime} \beta_{l},\right.
\end{aligned}
$$

com $\mathrm{E}\left(\beta_{1}\right)=\beta_{1}$ e supondo que $\mathrm{E}\left(\varepsilon_{1}\right)=0$.

Para identificar a contribuição das diferenças relativas aos grupos nos previsores para a diferença final resultante, a equação anterior pode ser rearranjada, por exemplo (Jahn, 2008, p. 2):

$$
\Delta=\left[\mathrm{E}\left(\mathrm{X}_{\mathrm{B}}\right)-\mathrm{E}\left(\mathrm{X}_{\mathrm{N}}\right)\right]^{\prime} \beta_{\mathrm{N}}+\mathrm{E}\left(\mathrm{X}_{\mathrm{N}}\right)^{\prime}\left(\beta_{\mathrm{B}}-\beta_{\mathrm{N}}\right)+\left[\mathrm{E}\left(\mathrm{X}_{\mathrm{B}}\right)-\mathrm{E}\left(\mathrm{X}_{\mathrm{N}}\right)\right]^{\prime}\left(\beta_{\mathrm{B}}-\beta_{\mathrm{N}}\right)
$$

Temos aqui um modelo de decomposição de três dobras (three-fold), em que o diferencial resultante é dividido em três partes: $\Delta=\mathrm{D}+\mathrm{C}+\mathrm{I}$, onde a primeira parte da soma, $\mathrm{D}=\left[\mathrm{E}\left(\mathrm{X}_{\mathrm{B}}\right)-\mathrm{E}\left(\mathrm{X}_{\mathrm{N}}\right)\right]^{\top} \beta_{\mathrm{N}}$, acrescenta à parte do diferencial o que é devido às diferenças dos grupos nos preditores (efeito das dotações). $O$ segundo componente, $C=E\left(X_{N}\right)^{\prime}\left(\beta_{B}-\beta_{N}\right)$, mede a contribuição das diferenças nos coeficientes, incluindo as diferenças no intercepto.

${ }^{6}$ Variável dummy com base na variável UF da Pnad, que compõe as regiões Sudeste e Sul (valor 1) e as demais regiões brasileiras, norte, nordeste e centro-oeste (valor 0).

${ }^{7}$ Grupo de dummies construído com base na variável v9048 (número de empregados no estabelecimento do trabalho principal, no mês de referência), assumindo as seguintes categorias: um; dois; três a cinco; seis a dez; mais de dez.

${ }^{8}$ Grupo de dummies construído com base na variável v9907 (código de atividade econômica), com as seguintes categorias: agrícolas; indústria de transformação; extrativismo; construção; comércio e reparação; alojamento e alimentação; educação, saúde e serviços sociais; outros serviços coletivos, sociais e pessoais; outras atividades econômicas. Entre empregadores estão ausentes as atividades de "serviços domésticos" e "administração pública" em 2014, embora ocorram eventualmente em outros anos. 
O terceiro elemento, $\mathrm{I}=\left[\mathrm{E}\left(\mathrm{X}_{\mathrm{B}}\right)-\mathrm{E}\left(\mathrm{X}_{\mathrm{N}}\right)\right]^{\prime}\left(\beta_{\mathrm{B}}-\beta_{\mathrm{N}}\right)$ é um termo de interação, remetendo ao fato de que existem diferenças em dotações e contribuições simultaneamente entre os dois grupos.

A decomposição, que será modelada pela equação destacada, é formulada do ponto de vista do grupo N, de trabalhadores negros, ou seja, as diferenças do grupo nos regressores são ponderadas pelos coeficientes do grupo $\mathrm{N}$ para determinar o efeito de dotação D. Em outras palavras, o componente D mede a mudança esperada na média dos rendimentos de negros, se estes contassem com os índices dos preditores do grupo dos brancos. Assim também para o componente $\mathrm{C}$, as diferenças nos coeficientes são ponderadas pelos níveis de predição do grupo de negros, ou seja, o componente mede a mudança esperada na média de rendimentos dos negros, se estes contassem com os coeficientes dos brancos.

A amostra de empregadores foi extraída mediante uma integração dos arquivos "pessoas" e "domicílios", que compõem os microdados da Pnad de 2014. Como a Pnad envolve uma amostragem complexa, os resultados a seguir compõem uma estimativa cuja base empírica não é mais qualquer empregador, mas apenas aqueles que são entrevistados em cada domicílio. A estimativa é calculada levando em conta os pesos das variáveis v4729 (peso da pessoa), v4618 (Strat - Identificação de estrato de município autorrepresentativo e não autorrepresentativo) e v4617 (UPA - Unidade primária de amostragem). ${ }^{9}$ Os resultados da decomposição de Oaxaca-Blinder aplicados aos rendimentos de empregadoras/es brancos e negros, segundo a Pnad de 2014, estão expressos e detalhados no quadro 1.

Os resultados mostram, no primeiro painel (diferencial), os valores esperados para as médias de rendimentos (em logaritmos naturais) de brancos (previsão 1) e negros (previsão 2), com base nos sete atributos observáveis como variáveis integrantes do modelo de regressão linear e sua diferença, ou seja, o total da discrepância racial de renda entre empregadores brancos e empregadores negros. Assim, há uma diferença geral de 0,33 (renda por hora em escala logarítmica) em favor do rendimento dos brancos, que corresponde, em escala exponencial, ${ }^{10}$ a uma diferença de 39\% (R \$ 5,56/h). Essa diferença é decomposta em três partes, conforme explicitado nos painéis subsequentes.

\footnotetext{
${ }^{9}$ A preparação dos dados para o emprego do método de decomposição foi realizada com base em recomendações de Ernesto Friedrich de Lima Amaral <http://www.ernestoamaral.com/ mq.html>, Jerônimo O. Muniz (UFMG) e Gilnei Costa Santos (UFV) em cursos de econometria ou métodos de análise quantitativa. O procedimento foi realizado no Stata/SE versão 11.0.

${ }^{10} \mathrm{O}$ rendimento médio em reais é convertido em escala logarítmica para se adequar melhor à análise de regressão. A reconversão dos valores em logaritmo para reais gera valores em escala exponencial. A média exponencial dos rendimentos representa valores com certo grau de subestimação quando comparada à média aritmética, mais usual quando tratamos de renda.
} 
Quadro 1. Empregadores - Decomposição para grupos de raça/cor (Brasil, 2014). Número de observações: 6.141 - Tamanho estimado da população: 3.625 .401 Grupos: 1 - brancos; 2 - negros

\begin{tabular}{|c|c|c|c|c|}
\hline Rendimento por hora & Coef. (B) (log) & b (exp) & $\mathbf{t}$ & $p>|t|$ \\
\hline \multicolumn{5}{|l|}{ Diferencial } \\
\hline - previsão 1 & 2,98 & 19,69 & 178,39 & 0,000 \\
\hline - previsão 2 & 2,65 & 14,13 & 127,30 & 0,000 \\
\hline - diferença & 0,33 & 1,39 & 12,45 & 0,000 \\
\hline \multicolumn{5}{|l|}{ Dotações } \\
\hline- sexo & $-0,01$ & 0,99 & $-2,85$ & 0,004 \\
\hline - escolaridade & 0,06 & 1,07 & 6,10 & 0,000 \\
\hline - idade & 0,04 & 1,04 & 2,92 & 0,003 \\
\hline - tempo de experiência de trabalho & $-0,01$ & 0,99 & $-1,35$ & 0,178 \\
\hline - localização geográfica & $-0,00$ & 0,99 & $-0,41$ & 0,682 \\
\hline - um empregado & omitida & & & \\
\hline - dois empregados & $-0,01$ & 0,99 & $-1,87$ & 0,061 \\
\hline - três a cinco empregados & $-0,01$ & 0,99 & -2.04 & 0,041 \\
\hline - seis a dez empregados & 0,02 & 1,02 & 4,29 & 0,000 \\
\hline - onze ou mais empregados & 0,06 & 1,06 & 6,10 & 0,000 \\
\hline - atividades agrícolas & omitida & & & \\
\hline - indústria de transformação & 0,01 & 1,01 & 1,38 & 0,169 \\
\hline - extrativismo & $-0,00$ & 1,00 & $-0,55$ & 0,582 \\
\hline - construção & $-0,05$ & 0,98 & $-2,08$ & 0,037 \\
\hline - comércio e reparação & 0,00 & 1,00 & 0,00 & 0,998 \\
\hline - alojamento e alimentação & $-0,00$ & 1,00 & $-0,90$ & 0,370 \\
\hline - transporte, armazenagem e comunicação & 0,00 & 1,00 & 0,27 & 0,786 \\
\hline - educaccão, saúde e serviços sociais & 0,04 & 1,04 & 3,57 & 0,000 \\
\hline - outros serviços coletivos, sociais e pessoais & $-0,00$ & 1,00 & $-0,76$ & 0,447 \\
\hline - outras atividades econômicas & 0,04 & 1,04 & 2,72 & 0,007 \\
\hline - Total & 0,19 & 1,20 & 8,96 & 0,000 \\
\hline \multicolumn{5}{|l|}{ Contribuições } \\
\hline- sexo & $-0,00$ & 1,00 & $-0,07$ & 0,946 \\
\hline - escolaridade & 0,11 & 1,11 & 1,39 & 0,164 \\
\hline - idade & 0,75 & 2,11 & 2,57 & 0,010 \\
\hline - tempo de experiência de trabalho & $-0,40$ & 0,67 & $-2,27$ & 0,023 \\
\hline - localização geoográfica & 0,01 & 1,01 & 0,55 & 0,579 \\
\hline - um empregado & omitida & & & \\
\hline - dois empregados & $-0,02$ & 0,98 & $-1,55$ & 0,122 \\
\hline - de três a cinco empregados & $-0,01$ & 0,99 & $-0,78$ & 0,434 \\
\hline - de seis a dez empregados & $-0,01$ & 0,98 & $-2,07$ & 0,038 \\
\hline - onze ou mais empregados & 0,01 & 1,00 & 1,01 & 0,313 \\
\hline - atividades agrícolas & omitida & & & \\
\hline - indústria de transformação & $-0,02$ & 0,98 & $-0,38$ & 0,705 \\
\hline - extrativismo & $-0,00$ & 1,00 & $-1,14$ & 0,256 \\
\hline - construção & $-0,02$ & 0,98 & $-0,36$ & 0,720 \\
\hline - comércio e reparação & $-0,03$ & 0,97 & $-0,17$ & 0,865 \\
\hline - alojamento e alimentação & $-0,00$ & 1,00 & 0,09 & 0,928 \\
\hline - transporte, armazenagem e comunicação & $-0,01$ & 0,98 & $-0,88$ & 0,380 \\
\hline - educação, saúde e serviços sociais & $-0,01$ & 0,99 & $-0,71$ & 0,478 \\
\hline - outros serviços coletivos, sociais e pessoais & 0,00 & 1,00 & 0,16 & 0,875 \\
\hline - outras atividades econômicas & $-0,00$ & 1,00 & $-0,09$ & 0,927 \\
\hline - constante & 0,24 & 0,79 & $-0,55$ & 0,580 \\
\hline - Total & 0,10 & 1,10 & 3,86 & 0,000 \\
\hline \multicolumn{5}{|l|}{ Interações } \\
\hline - idade & 0,03 & 1,03 & 2,23 & 0,026 \\
\hline - de seis a dez empregados & $-0,01$ & 1,00 & $-0,05$ & 0,019 \\
\hline - outros fatores & 0,03 & $\sim 1,00$ & $-0,08$ & $>=0,05$ \\
\hline - Total & 0,05 & 1,05 & 2,10 & 0,0351 \\
\hline
\end{tabular}


O segundo painel (dotações) demonstra que, do total da diferença entre brancos e negros, $19 \%(\mathrm{R} \$ 1,05 / \mathrm{h})$ decorre de diferenças nas características produtivas dos dois grupos. É o mesmo que dizer que, se os empregadores negros tivessem as características produtivas dos empregadores brancos, seu rendimento seria, em média, 19\% (R \$ 1,05 por hora) superior. Esta é a parte da diferença que é explicada pelas variáveis inseridas no modelo. O terceiro painel (contribuições) aponta que $10 \%(\mathrm{R} \$ 0,56 / \mathrm{h})$ da vantagem dos brancos decorre de sua remuneração superior em relação aos negros mesmo quando as características produtivas são controladas. Neste caso, o comportamento dos coeficientes simula o que pode ser interpretado como a discriminação no mercado de trabalho. Essa medida expressa a mudança esperada no logaritmo do rendimento/hora de empregadores negros pela contribuição contrafactual dos coeficientes dos empregadores brancos em suas características. Esta contribuição, que totalizaria um acréscimo de $10 \%$ ( $\mathrm{R} \$ 0,56$ por hora) no rendimento esperado de negros (caso contassem com os coeficientes dos brancos), é que caracteriza propriamente um efeito da discriminação racial, ou seja, a parte da diferença que não é explicada pelas características produtivas dos grupos raciais que constituem elementos observáveis da estrutura econômica e social em que a trajetória do empregador está inserida. O pequeno acréscimo de 5\% (R \$ 0,28/h) que aparece no quarto painel (Interações) decorre do efeito simultâneo das diferenças nas dotações e coeficientes.

A análise da decomposição revela que as principais variáveis responsáveis pelo diferencial são aquelas ligadas ao modelo minceriano para explicação do valor dos rendimentos do trabalho: escolaridade e tempo de experiência de trabalho. Quase um quinto do diferencial entre médias geométricas de rendimentos de negros e de brancos $(19 \%$, valor do componente de "dotações" no quadro 1) pode ser creditado a diferenças de características pessoais (idade, anos de experiência laboral, nível de escolaridade e sexo) ou características econômicas, como o número de empregados ou o tipo de atividade econômica desenvolvida. A maioria dessas contribuições implica rendimentos relativamente maiores aos brancos, caso fixemos os demais fatores de controle: idade (4\%), escolaridade (6\%), número de empregados acima de 5 (8\%). Dentre as atividades econômicas, sua contribuição não é estatisticamente significante para explicar o diferencial, com exceção de dois grupos: "educação, saúde e serviços sociais" (4\%) e "outras atividades econômicas" (4\%). Há, por outro lado, dois fatores cujos valores incrementam os rendimentos de negros: anos de experiência laboral (1\%) e sexo (1\%). No que se refere à experiência no trabalho, o resultado reforça o que a literatura sobre desigualdades raciais constata, ou seja, de que os negros começam a 
trabalhar mais cedo, por condicionantes históricos e sociais com origem na dominação colonialista e escravista. O resultado para esse preditor indica que, se negros e brancos empregadores tiverem o mesmo rendimento e mantendo o valor de todos os demais preditores para ambos, empregadores brancos terão em média ${ }^{11} 4 \%$ mais idade que os negros e $6 \%$ mais em relação à escolaridade. No caso de sexo, o diferencial em favor de negros ocorre, mas remete a homens negros, conforme comentaremos a seguir, o que indica mais, neste caso, uma desigualdade de gênero do que uma diferença decorrente da característica de cor ou raça.

A decomposição indica que $10 \%$ do diferencial total de rendimentos entre empregadores brancos e negros (valor do componente de "contribuições" no quadro 1) pode ser atribuído à discriminação, ainda que também possa incorporar outros fatores não observáveis relacionados à organização do trabalho (como qualidade do treinamento) ou à subjetividade, como capacidade de liderança, disposição para a comunicação e o relacionamento no local de trabalho (Cacciamali e Tatei, 2013). As variáveis influentes na composição do valor dos rendimentos podem alterar o sentido e a magnitude de sua influência, bem como se tornarem estatisticamente não significantes. A escolaridade, por exemplo, não é estatisticamente significante quando deslocada de sua função estrutural na composição dos rendimentos. Já a idade explica $75 \%$ do diferencial correspondente à discriminação, constituindo o fator mais influente relacionado a uma diferença de rendimentos favorável a brancos. Em outras palavras, se considerarmos as características produtivas dos negros controladas, sua idade seria desvalorizada em $75 \%$. Por outro lado, a experiência de trabalho contaria relativamente em seu favor, $40 \%$ em relação aos brancos. Porém a escolaridade mais alta possivelmente correlacionada com a idade não é significante nesse componente; assim, valorizar a experiência de trabalho dos negros como empregadores possivelmente implica dar relevo ao tempo empregado em setores ocupacionais de menor valor agregado, como reparação e manutenção ou alimentação e alojamento. O tempo de experiência no trabalho interfere positivamente em favor dos empregadores negros, como dotação (1\%) e também como coeficiente, relacionado à discriminação (40\%). Entretanto, a magnitude dessa influência é suplantada pela contribuição, em sentido contrário, de variáveis como idade, escolaridade, maior número de empregados e trabalho em setores mais qualificados, originando um total de $26 \%$ em desfavor dos negros nas dotações e $85 \%$ no mesmo sentido decorrente da discriminação. O tempo de experiência, como preditor, apresenta uma

\footnotetext{
${ }^{11}$ Trata-se da média geométrica e não da média aritmética.
} 
peculiaridade que não fica visível no quadro 1 , mas desponta nas equações de regressão linear de empregadores negros e brancos, que alimentam o modelo de decomposição OB. Nos dois grupos essa variável diminui a diferença entre os rendimentos ${ }^{12} \mathrm{e}$ isso pode estar relacionado ao fato de que, quando aumenta o tempo de trabalho não há diferença significativa em relação ao que ocorre nas décadas iniciais da carreira, em que um aumento na idade geralmente é acompanhado também de um aumento na escolaridade ou na qualificação.

Outro resultado importante derivado da decomposição é ausência de contribuição da variável "localização geográfica" na composição do valor de rendimentos e também, de modo geral, o baixo valor (5\%) do termo relativo à "interação", indicando baixa influência de efeitos simultâneos entre as diferenças e os próprios coeficientes e dotações de negros e brancos.

O quadro 2 demonstra a permanência do tipo de contribuição da discriminação e da influência dos regressores na estimação do valor de rendimentos de brancos e negros. Embora a magnitude da diferença tenha sido maior em 2008, 2009 e 2013, nos seis anos considerados o sentido da influência é o mesmo, ou seja, tanto as características "visíveis" que contribuem para o resultado quanto os fatores não explicados das regressões, dos quais sustentamos que o principal é a discriminação, influem negativamente no valor dos rendimentos dos negros. Vale ressaltar que, em 2009 e 2011 a influência da discriminação (contribuição dos coeficientes) foi superior (17\% em 2009 e $19 \%$ em 2011) à influência das variáveis sistêmicas (16\% em 2009 e apenas $6 \%$ em 2011). Nesses anos a discriminação racial suplantou as características econômicas e pessoais (já que, em todos os anos examinados, a variável de localização geográfica não produziu resultados estatisticamente significantes). Contudo, a apresentação desses quadros da decomposição em anos diferentes não foi realizada com os cuidados técnicos e metodológicos que caracterizariam propriamente uma análise longitudinal e também não temos condições, neste artigo, de especular sobre as variações encontradas em 2009 e 2011.

É conveniente compararmos a decomposição OB dos rendimentos de grupos de brancos e negros com a decomposição em grupos de homens e mulheres, considerando outro tipo de discriminação também relevante para empregadores (quadro 3).

\footnotetext{
${ }^{12}$ Não reproduzimos aqui os resultados das regressões do modelo de decomposição OB para os dois grupos, de brancos e negros. No entanto, os valores do coeficiente angular $(-0,021701$ para brancos e 0,007993 para negros) demonstram a relação inversa entre o tempo de experiência e os rendimentos de brancos e negros, ainda que o resultado deste não tenha obtido significância estatística para os negros.
} 


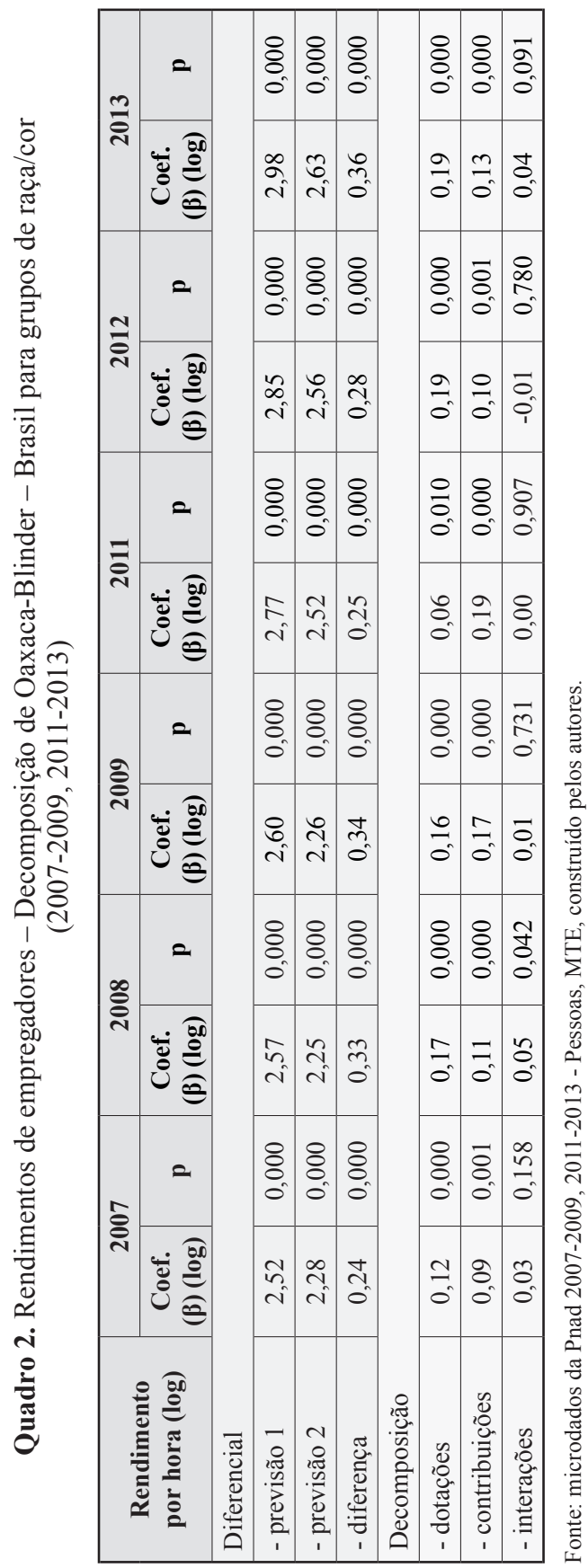


Quadro 3. Rendimentos de empregadores - Decomposição de Oaxaca-Blinder para grupos de sexo (Brasil, 2014).

Número de observações: 6.141 - Tamanho estimado da população: 3.625 .401 Grupos: 1 - mulheres $=0 ; 2$ - homens $=1$

\begin{tabular}{|l|c|c|c|c|c|c|}
\hline $\begin{array}{c}\text { Rendimento } \\
\text { por hora }\end{array}$ & $\begin{array}{c}\text { Coef. } \\
(\boldsymbol{\beta})(\mathbf{l o g})\end{array}$ & $\mathbf{b}(\mathbf{e x p )}$ & $\mathbf{t}$ & $\mathbf{p}>|\mathbf{t}|$ & \multicolumn{2}{|c|}{$\begin{array}{c}\text { Int. confiança } \\
\mathbf{9 5 \%}(\mathbf{e x p})\end{array}$} \\
\hline Diferencial & & & & & & \\
\hline - previsão 1 & 2,82 & 16,86 & 113,38 & 0,000 & 16,06 & 17,70 \\
\hline - previsão 2 & 2,90 & 18,14 & 182,40 & 0,000 & 17,58 & 18,71 \\
\hline - diferença & $-0,07$ & 0,93 & $-2,47$ & 0,001 & 0,88 & 0,98 \\
\hline Decomposição & & & & & & \\
\hline - dotações & 0,09 & 1,10 & 4,80 & 0,000 & 1,06 & 1,14 \\
\hline - contribuições & $-0,14$ & 0,87 & $-4,20$ & 0,000 & 0,81 & 0,93 \\
\hline - interações & $-0,02$ & 0,98 & $-0,85$ & 0,394 & 0,93 & 1,03 \\
\hline
\end{tabular}

Fonte: microdados da Pnad 2014 - Domicílios e Pessoas, MTE, construído pelos autores.

A discriminação de gênero para empregadoras é, segundo os coeficientes da decomposição realizada, mais expressiva que a de raça/cor, influindo em $14 \%$ em desfavor das mulheres para a diferença encontrada (em relação a raça/ cor a contribuição é de $10 \%$ ). Porém, a contribuição dos fatores produtivos associados ao rendimento no modelo empregado incide em outro sentido, favorável às mulheres ( $9 \%$ no item de dotações). Como também neste caso a contribuição de interações não é estatisticamente significante, resulta uma diferença na estimativa dos rendimentos pelo modelo aplicado aos grupos de homens e mulheres de 7\% em favor dos homens. Comparando as estimativas de rendimentos (média geométrica) pelos modelos de regressão aplicados a grupos de raça $(14,13$ reais/hora para negros e 19,69 reais/hora para brancos) e a grupos de gênero (16,86 reais/hora para mulheres e 18,14 reais/ hora para homens) notamos que as contribuições de fatores correlacionados com diferenças de raça/cor tendem a produzir rendimentos mais altos para brancos, mais até do que para homens em relação a fatores correlacionados com diferenças de sexo. Em outras palavras, a conjunção de características "visíveis" relacionadas ao capital humano, como escolaridade e experiência e fatores "invisíveis" supostamente relacionados à discriminação origina valores de rendimentos maiores para homens e brancos em relação a grupos de raça/ cor e sexo. Entretanto, no caso de grupos de cor, a amplitude interna das diferenças é maior. A análise das duas decomposições nos leva a considerar que, na conjuntura atual que contextualiza a atividade laboral de empregadores/ 
as homens e mulheres no Brasil, os fatores estruturais, já pressionados pela ação de grupos feministas e políticas de equidade, tendem a contrapor uma desvalorização do trabalho feminino, relativo ao tipo e à posição na ocupação, originalmente muito grande, numa diferença que, embora ainda desfavorável às mulheres, é menor do que a que ocorre entre rendimentos de brancos $\mathrm{e}$ negros. Neste caso, as diferenças culturais e originárias no mundo do trabalho são proeminentes, embora encontrem resistência, principalmente diante das exigências de isonomia e políticas recentes de equidade racial, mas as diferenças que provêm dos próprios fatores sistêmicos, principalmente a escolaridade relativamente deficitária, o ingresso precoce na esfera laboral e a "herança" sucessiva de posições ocupacionais socialmente menos valorizadas, têm grande peso. O resultado é que a discriminação no trabalho se soma à desigualdade de classe e raça, enquanto que, no caso de gênero, a desigualdade de classe não é estruturalmente relacionada a diferenças de sexo e, por isso estas são atenuadas pela influência positiva dos fatores sistêmicos, ainda que as desigualdades de gênero sejam histórica e culturalmente produtoras de diferenças no mundo do trabalho, em termos de ocupações e rendimentos.

\section{Considerações finais}

Podemos concluir que boa parte do diferencial de rendimentos entre brancos e negros que se encontram na posição de empregador devese às influências das variáveis consideradas fundamentais na teoria do capital humano, ou seja, escolaridade e experiência de trabalho, embora características produtivas, como número de empregados, também contribuam significativamente. Entretanto, o resíduo da diferença não explicado pelas características pessoais e econômicas remete a $10 \%$ do diferencial de rendimentos entre brancos e negros empregadores. Considerando que esse montante pode conter também a influência de variáveis não contidas no modelo, estima-se que boa parte dele se deve a alguma influência da discriminação racial que, historicamente incorporada às relações sociais como um todo e, em especial, ao mercado de trabalho, faz com que empregadores negros não alcancem os mesmos rendimentos de empregadores brancos, mesmo quando suas características produtivas são semelhantes.

Para o entendimento adequado dos resultados, é preciso levar em conta a tese - sustentada fortemente pela literatura sobre desigualdade no mercado de trabalho - de que a discriminação racial se efetiva em diferentes etapas da vida dos negros, desde a sua origem social, passando pela escolarização, pela transição da escola para o trabalho, pela inserção ocupacional, até chegar aos rendimentos. Assim, muitos dos efeitos da discriminação já se colocaram como 
barreiras para que os negros pudessem alcançar a posição de empregador. Amparados pelos resultados da literatura sociológica, que demonstram que os brancos são maioria nos estratos ocupacionais mais elevados, podemos concluir que a segmentação do mercado de trabalho exerce um efeito anterior ao da discriminação direta que atinge os negros que se encontram na classe dos empregadores, apresentando-se, portanto, na forma de efeitos indiretos sobre os seus rendimentos. Os efeitos da segmentação se apresentam disseminados no território nacional, o que explica a falta de significância da variável relativa à região de inserção dos empregadores.

A influência da discriminação e a das variáveis sistêmicas alternam-se como fundamentais na construção da desigualdade durante os anos recentes, o que pode ser verificado na análise longitudinal. Apesar disso, é possível verificar uma permanência da discriminação, notada pelo nível considerável do resíduo ao longo dos anos.

Uma das conclusões mais interessantes e que ilustram a complexidade dos efeitos das características individuais e da discriminação sobre os rendimentos pode ser obtida na comparação entre os resultados da decomposição por raça e da decomposição por sexo. Se, para os negros, tanto os efeitos dos coeficientes (discriminação) quanto os efeitos das dotações (características pessoais) influem negativamente, para as mulheres os efeitos dos coeficientes (discriminação) influem negativamente, porém os efeitos das dotações (características pessoais) influem positivamente. Este resultado está de acordo com os achados das pesquisas mais recentes que apontam que, a partir do final da década de 1970 o nível de escolaridade das mulheres ultrapassou o verificado para os homens. Desde então, as mulheres vêm experimentando um incremento médio de suas características produtivas - a despeito das diferenças entre mulheres brancas e mulheres negras. Disto resulta que, no interior da classe dos empregadores, a desigualdade atinge tanto as mulheres quanto os negros. Contudo, como demonstra o resultado da decomposição, a parte do diferencial de rendimentos que pode ser atribuída à discriminação em favor dos homens em relação às mulheres é maior do que a parte do diferencial de rendimentos que pode ser atribuído à discriminação em favor dos brancos em relação aos negros (há que se considerar, novamente, a especificidade das mulheres negras, grupo historicamente desfavorecido em todas as posições de classe). Isto demonstra que a desigualdade entre brancos e negros, decorrente da discriminação no mercado de trabalho, é significativa não apenas quando se compara a distribuição por raça entre os grupos ocupacionais e as posições de classe, mas também dentro dos grupos ocupacionais e dentro das classes. Consideramos significativo o fato de que, entre os empregadores, a 
desigualdade racial e de gênero demonstra a mesma tendência verificada entre os empregados, dado que este último grupo tende a ser mais heterogêneo, enquanto o primeiro se caracteriza por requisitos mais específicos.

\section{Referências}

ATKINSON, A. Income and inequality in OECD countries: data and explanation. CESIfo Economic Studies, v. 49, n. 4, p. 479-513, $2003<10.1093 /$ cesifo/49.4.479>.

BECKER, Gary Stanley. Investment in human capital: a theoretical analysis. Journal of Political Economy, v. 70, n. 5, parte 2, p. 9-49, 1962.

BLINDER, Alan Stuart. Wage discrimination: reduced form and structural estimates. The Journal of Human Resources, v. 8, n. 4 p. 436-455, $1973<10.2307 / 144855>$.

CACCIAMALI, Maria Cristina; TATEI, Fábio. Género y salarios de la fuerza de trabajo calificada en Brasil y México. Problemas del Desarrollo, v. 44, n. 172, p. 53-79, 2013.

CARVALHO, A. P.; NÉRI, M. C.; SILVA, D. B. Diferenciais de salários por raça e gênero: aplicação dos procedimentos de Oaxaca e Heckman em pesquisas amostrais complexas. Caxambu, 2006. 15. Encontro de Estudos Populacionais (Abep) <http:// www.abep.nepo.unicamp.br/encontro2006/docspdf/ABEP2006_695.pdf>.

CARD, David; DINARDO, John E. Skill-biased technological change and rising wage inequality: some problems and puzzles. Journal of Labor Economics, v. 20, n. 4 , p. $733-783,2002<10.1086 / 342055>$.

DAHRENDORF, R. Class and class conflict in industrial society. In: David Grusky (org.). Social stratification: race, class and gender in sociological perspective. 4. ed. Colorado: Westview Press, 2014. p. 143-149.

DIPRETE, Thomas A. What has sociology to contribute to the study of inequality trends? A historical and comparative perspective. American Behavioral Scientist, v. 50, n. 5, p. 603-618, $2007<10.1177 / 0002764206295009>$.

ERIKSON, Robert; GOLDTHORPE, John H. The constant flux: a study of class mobility in industrial societies. Oxford: Claredon Press, 1992.

ERIKSON, Robert, GOLDTHORPE, John H. Intergenerational inequality: a sociological perspective. Journal of Economic Perspectives, v. 16, n. 3, p. 31-44, 2002 $<10.1257 / 089533002760278695>$.

GRUSKY, Dadid. The past, present, and future of social inequality. In: David Grusky (org.). Social stratification: class, race and gender in sociological perspective. Boulder: Westview, 2001. p. 3-51.

GUIMARÃES, Nadya Araújo. Os desafios da equidade: reestruturação e desigualdades de gênero e raça no Brasil. Cadernos Pagu, n. 17-18, p. 237-266, $2002<10.1590$ / S0104-83332002000100009>.

JANN, Ben. The Blinder-Oaxaca decomposition for linear regression models. The Stata Journal, v. 8, n. 4, p. 453-479, 2008.

HASENBALG, C. Discriminação e desigualdades raciais no Brasil. Rio de Janeiro: Graal, 1979. 
HASENBALG, C.; SILVA, N. V. Estrutura social, mobilidade e raça. Rio de Janeiro: Vértice, 1988.

HASENBALG, C.; SILVA, N. V.; LIMA, M. Cor e estratificação social no Brasil. Rio de Janeiro: ContraCapa, 1999.

HASENBALG, C.; SILVA, N. V. (orgs.). Origens e destinos: desigualdade sociais ao longo da vida. Rio de Janeiro: Topbooks, 2003.

LIMA, M. Serviço de branco, serviço de preto: um estudo sobre cor e trabalho no Brasil urbano. Iuperj: Rio de Janeiro, 2001. Tese de doutorado em Sociologia.

LIMA, Márcia. "Raça" e pobreza em contextos metropolitanos. Tempo Social, v. 24, n. $2,2012<10.1590 / \mathrm{S} 0103-20702012000200012>$.

MEDEIROS, M. O que faz os ricos ricos? Um estudo sobre os fatores que determinam a riqueza. Universidade de Brasília. Brasília, 2003. Tese de Doutorado em Sociologia.

MINCER, J. Schooling, experience, and earnings. New York: NBER, 1974.

MYLES, John. Where have all the sociologists gone? Explaining income inequality. The Canadian Journal of Sociology, v. 28, n. 4, p. 551-559, $2003<10.2307 / 3341842>$.

OAXACA, Ronald. Male-female wage differentials in urban labor markets. International Economic Review, v. 14, n. 3, p. 693-709, $1973<10.2307 / 2525981>$.

OSÓRIO, R. G. A mobilidade social dos negros brasileiros. Ipea: Texto para discussão 1033, 2004.

OSÓRIO, R. G. A desigualdade racial de renda no Brasil: 1976-2006. Brasília: Universidade de Brasília, 2009. Tese de doutorado em Sociologia.

PIKETTY, Thomas, SAEZ, Emmanuel. Income inequality in the United States 1913-1998. Quarterly Journal of Economics, v. 118, n. 1 p. 1-41, $2003<10.1162$ / $00335530360535135>$.

RIBEIRO, C. A. C. Desigualdade de oportunidades no Brasil. Belo Horizonte: Argvmentvm, 2009.

ROCHA, E. F. O negro no mundo dos ricos: um estudo sobre a disparidade racial de riqueza no Brasil com os dados do censo demográfico de 2010. Universidade de Brasília: Brasília, 2015. Tese de doutorado em Sociologia.

SANTOS, José Alcides Figueiredo. Efeitos de classe na desigualdade racial no Brasil. Dados, v. 48, n. 1, p. 21-65, $2005<10.1590 /$ S0011-52582005000100003>.

SANTOS, José Alcides Figueiredo. Classe social e desigualdade de gênero no Brasil. Dados, v. 51 n. 2, p. 353-402, $2008<10.1590 /$ S0011-52582008000200005>.

SANTOS, José Alcides Figueiredo. A interação estrutural entre a desigualdade de raça e de gênero no Brasil. Revista Brasileira de Ciências Sociais, v. 24, n. 70, p. 37-60, $2009<10.1590 /$ S0102-69092009000200003>.

SCALON, Celi. Ensaios de estratificação. Belo Horizonte: Argumentum, 2009.

SOARES, S. S. D. O perfil da discriminação no mercado de trabalho: homens negros, mulheres brancas e mulheres negras. Ipea, Texto para discussão n. 769, 2000.

SØRENSEN, Aage B. Toward a sounder basis for class analysis. American Journal of Sociology, v. 105, n. 6, p. 1523-1558, $2000<10.1086 / 210463>$. 
THERBORN, G. Sexo e poder: a família no mundo, 1900-2000. São Paulo: Editora Contexto, 2006.

TILLY, Charles. Durable inequality. Berkeley: University of California Press, 1999.

WRIGHT, Erik Olin; PERRONE, Luca. Marxist class categories and income inequality. American Sociological Review, v. 42, n. 1, p. 32-55, $1977<10.2307 / 2117730>$.

WRIGHT, E. O. Class structure and income determination. New York: Academic Press, 1979.

WRIGHT, E. O. Intersections and interactions: thinking about the relationship between class and other forms of inequality. Lecture 7, Sociology 298, $2002<\mathrm{https}$ :// www.ssc.wisc.edu/ wright/SOC621/298lec7.pdf> (18 mar. 2015).

WRIGHT, E. O. Approaches to class analysis. New York: Cambridge University Press, $2005<10.1017 /$ CBO9780511488900>.

Autor correspondente:

Jordão Horta Nunes

Rua 88-B, 678, ap. $502-$ S. Sul

74085-030 Goiânia, GO, Brasil

Recebido em: 24 dez. 2015

Aprovado em: 27 abr. 2016 